\title{
Ways to improve the confinement of fast ions in stellarators by RF waves: general analysis and application to Wendelstein $7-\mathrm{X}$
}

\author{
Ya.I. Kolesnichenko ${ }^{1}$, V.V. Lutsenko ${ }^{1}$, T.S. Rudenko ${ }^{1}$, \\ P. Helander ${ }^{2}$ \\ ${ }^{1}$ Institute for Nuclear Research, Prospekt Nauky 47, Kyiv 03680, Ukraine \\ ${ }^{2}$ Max-Planck-Institut für Plasmaphysik, Wendelsteinstraße 1, D-17491, Greifswald, \\ Germany
}

\begin{abstract}
A possibility to improve the confinement of energetic ions in stellarators by transforming trapped particles into passing ones using RF waves is investigated. It is concluded that waves with frequencies around the ion gyrofrequency or lower can, in principle, be used for this purpose if certain requirements are satisfied. The suitability of the W7-X ICRH system is considered, and other means for achieving fast-ion confinement are discussed.
\end{abstract}

1 February 2017

PACS numbers:

Submitted to: Nuclear Fusion 


\section{Introduction}

One of the most difficult challenges facing a stellarator fusion reactor is the confinement of fast ions, in particular alpha particles. Several methods are available for tailoring the magnetic field to optimize orbit confinement [1], and one of them (so-called quasiisodynamicity or an approximation thereof) has been employed in Wendelstein 7-X [2,3], in related reactor studies [4], and in more recent stellarator optimizations [5]. In these devices, the energetic ions are well confined provided that $\beta$ - the ratio of the plasma pressure $(p)$ to the magnetic field pressure $\left(B^{2} / 8 \pi\right)$ - is sufficiently high, $\beta \approx 5 \%[2,3]$. The physical mechanism responsible for the confinement improvement is associated with the poloidal magnetic drift of the fast ions. When $\beta$ is high enough, this drift reverses, leading to closed contours of the second adiabatic invariant $\left(\mathcal{J}=\oint v_{\|} d l\right)$. In other words, the particle radial drift averages to zero and constant-B contours close poloidally at high $\beta$. One says that the system in this case is quasi-isodynamic [6]. Unfortunately, quasiisodynamicity cannot be perfect. Therefore, orbit losses of some particles or collisionless diffusion of transitioning particles is inevitable [7]. These particles undergo collisionless transitions from locally trapped to locally passing and vice versa, which leads to a random walk across the magnetic field.

In the first experiments with neutral beam injection (NBI) on W7-X, $\beta$ will be relatively low because only half the NBI beam lines will be operative. For this reason, a considerable fraction of the energetic ions produced by NBI will be lost [3].

These circumstances provide the motivation for the present work. The purpose is to preliminarily investigate whether the confinement of energetic ions can be improved by using radiofrequency $(\mathrm{RF})$ waves to modify their orbits. The basic idea is to transform trapped particles, which may be not confined, into passing ones by carefully tailoring the injection of RF power into the plasma. (In any stellarator with good magnetic surfaces, the orbit-averaged radial drift of passing particles vanishes to lowest order in a gyroradius explansion and these particles are therefore well confined [1].) The required orbit transformation can be produced by pitch-angle scattering and/or radial displacements caused by the RF field. If the energy of the ions is high enough, $\mathcal{E} \gg \mathcal{E}_{c} \sim\left(M_{i} / M_{e}\right)^{1 / 3} T_{e}$ (where $T$ is the temperature, $M$ is the particle mass, and the subscripts " $\mathrm{e}$ " and "i" label electrons and ions, respectively), the pitch-angle scattering of these ions caused by Coulomb collisions is relatively weak and the main effect of collisions on the energetic ions is to slow them down. This implies that after any RF-induced orbit transformation, a typical energetic ions will remain passing until its energy drops to $\mathcal{E}_{c}$, and it will thus transfer most of its energy to the plasma (mainly, to electrons) before being completely thermalized or lost.

In experiments on the Wendelstein 7-AS stellarator, the NBI produced mainly passing ions with energies $\mathcal{E} \gg \mathcal{E}_{c}$, which were well confined, but in W7-X the injection energy is not far above $\mathcal{E}_{c}$, and pitch-angle scattering is more important. Although most NBI ions are not born on poorly confined trapped orbits, some of them will be scattered onto such orbits, and it is therefore desirable to improve their confinement. As we shall 
see, however, this is difficult with the existing RF system.

The structure of the article is as follows. Basic relations required for understanding the problem are presented in section 2. This section includes consideration of (i) conditions for wave-induced increases of the longitudinal particle velocity and the concomitant transformation of trapped orbits into passing ones, which follows the characteristics of a quasilinear kinetic equation; (ii) wave polarization; and (iii) resonances of the wave-particle interaction. Section 3 is devoted to the application to W7-X: the maximum pitch angle of trapped injected particles are evaluated, an analysis is carried out of the possible use of waves generated by the W7-X RF antenna, and possibilities for using lower-frequency-waves are discussed. Section 4 summarizes and discusses the results obtained. Appendix A analyses the characteristics of energetic ions produced by NBI in W7-X, including the superbanana motion of particles with pitch angles in the range of transition orbits. The effects of the neoclassical radial electric field on this motion is also considered.

\section{Basic relations}

\subsection{Quasilinear evolution as a tool for orbit transformation}

Formally, quasilinear diffusion of particles in a wave field occurs by wave-particle interaction at an infinite number of cyclotron harmonics $\omega=l \omega_{B}$, with $\omega_{B}$ the particle gyrofrequency and $l$ an integer, see e.g. [8-10]. However, during ion cyclotron resonance heating $(\mathrm{ICRH})$ typically only the low- $l$ terms contribute substantially to the quasilinear diffusion coefficient $(D)$ because of restrictions imposed by the resonance condition and because the Larmor radius $\left(\rho_{\perp}\right)$ can be small compared to the transverse wavelength. In addition, the difference between cyclotron harmonics $\left(\omega_{B}\right)$ is comparable to the wave frequency $(\omega)$ when $\omega \sim l \omega_{B}$ with $l \sim 1$, and different particles interact with the waves through resonances with different $l$. Therefore, it is reasonable to assume that resonances associated with different cyclotron harmonics do not overlap. We can thus restrict our attention to the quasilinear evolution associated with a single harmonic, either $l \sim 1$ or $l=0$.

The magnetic moment is not conserved when $\omega \sim \omega_{B}$, and the kinetic energy of the motion perpendicular to the magnetic field $\left(\mathcal{E}_{\perp}\right)$ thus changes when $l \neq 0$. As follows from the quasilinear theory, the change of $\mathcal{E}_{\perp}$ is related to the parallel kinetic energy $\left(\mathcal{E}_{\|}\right)$by

$$
\frac{d \mathcal{E}_{\|}}{d \mathcal{E}_{\perp}}=\frac{\omega}{l \omega_{B i}}-1
$$

whose solution (characteristics of the quasilinear equation) is

$$
\mathcal{E}_{\|}-\left(\frac{\omega}{l \omega_{B i}}-1\right) \mathcal{E}_{\perp}=\text { const }
$$

Here the inhomogeneity of the magnetic field is temporarily neglected but will be included in the analysis later. Note that equation (1) can be obtained also by using 
the symmetry properties of the resonant term in the particle Lagrangian, like this was done, e.g., in reference [11].

In particular, $\mathcal{E}_{\|} \approx$ const during ICRH with $\omega \approx l \omega_{B i}$. This is a drawback of ICRH because it means that this form of heating is usually accompanied by a decrease of the particle pitch $v_{\|} / v$, with $\mathbf{v}$ the particle velocity. As a result, a population of the energetic trapped ions with wide orbits and poor confinement tends to be produced.

In experiments, ICRH indeed produces high-energy tails in the distribution function of the heated ions. For instance, a non-Maxwellian tail consisting of $\alpha$-particles with energies up to several $\mathrm{MeV}$ is routinely observed in the JET tokamak [12]. This and many other experiments have demonstrated that ICRH can serve as a source of energetic ions suitable to study the physics of fusion products and other fast ions. In contrast, our purpose is to convert trapped fast ions into passing ones, with a minimum increase of the particle energy.

Quasilinear evolution with $l \neq 0$ associated with the absorption of wave power $(d \mathcal{E}>0)$ does not necessarily lead to an increase of $\mathcal{E}_{\perp}$, and evolution with $l=0$ evolution conserves $\mathcal{E}_{\perp}$. To understand why, it is useful to write the increase of the total energy of a resonant ion as

$$
d \mathcal{E}_{\|}+d \mathcal{E}_{\perp}>0
$$

Because of (1), this equation implies

$$
\frac{\omega}{l \omega_{B i}} d \mathcal{E}_{\perp}>0 \text {. }
$$

If we take $\omega>0$ it thus follows that $\mathcal{E}_{\perp}$ increases if $l>0$ but drops if $l<0$ and stays constant if $l=0$.

In order to see whether and when the RF heating can result in orbit transformations, let us introduce the pitch variable $\lambda=\mu_{p} B_{0} / \mathcal{E}$, where $\mu_{p}$ is the particle magnetic moment and $B_{0}$ the equilibrium magnetic field strength. (When the inhomogeneity of $B_{0}$ is later taken into account, $B_{0}$ will be replaced by its magnitude at a certain point, see a paragraph before equation (7) and the last sentence in this subsection). In this variable, equation (1) becomes

$$
\frac{d \lambda}{l \lambda_{*}-\lambda}=\frac{d \mathcal{E}}{\mathcal{E}}
$$

which leads to the characteristics

$$
\mathcal{E}\left(\lambda-l \lambda_{*}\right)=\text { const },
$$

where $\lambda_{*}=\omega_{B i} / \omega$. We thus conclude that any increase in energy is accompanied by a reduction of $\lambda$, which in an inhomogeneous field can result in the transformation of trapped particles to passing ones if either $l \leq 0$ or if $l>0$ and $\lambda_{*}<\lambda_{\text {max }}^{\text {pass }} / l<\lambda^{\text {tr }} / l$, where superscripts "tr" and "pass" refer to the trapped and passing particle populations, respectively.

Figure 1 demonstrates this relation for the W7-X high-mirror configuration. It is seen in this figure that an increase of the particle energy by $10-20 \%$ is sufficient for 

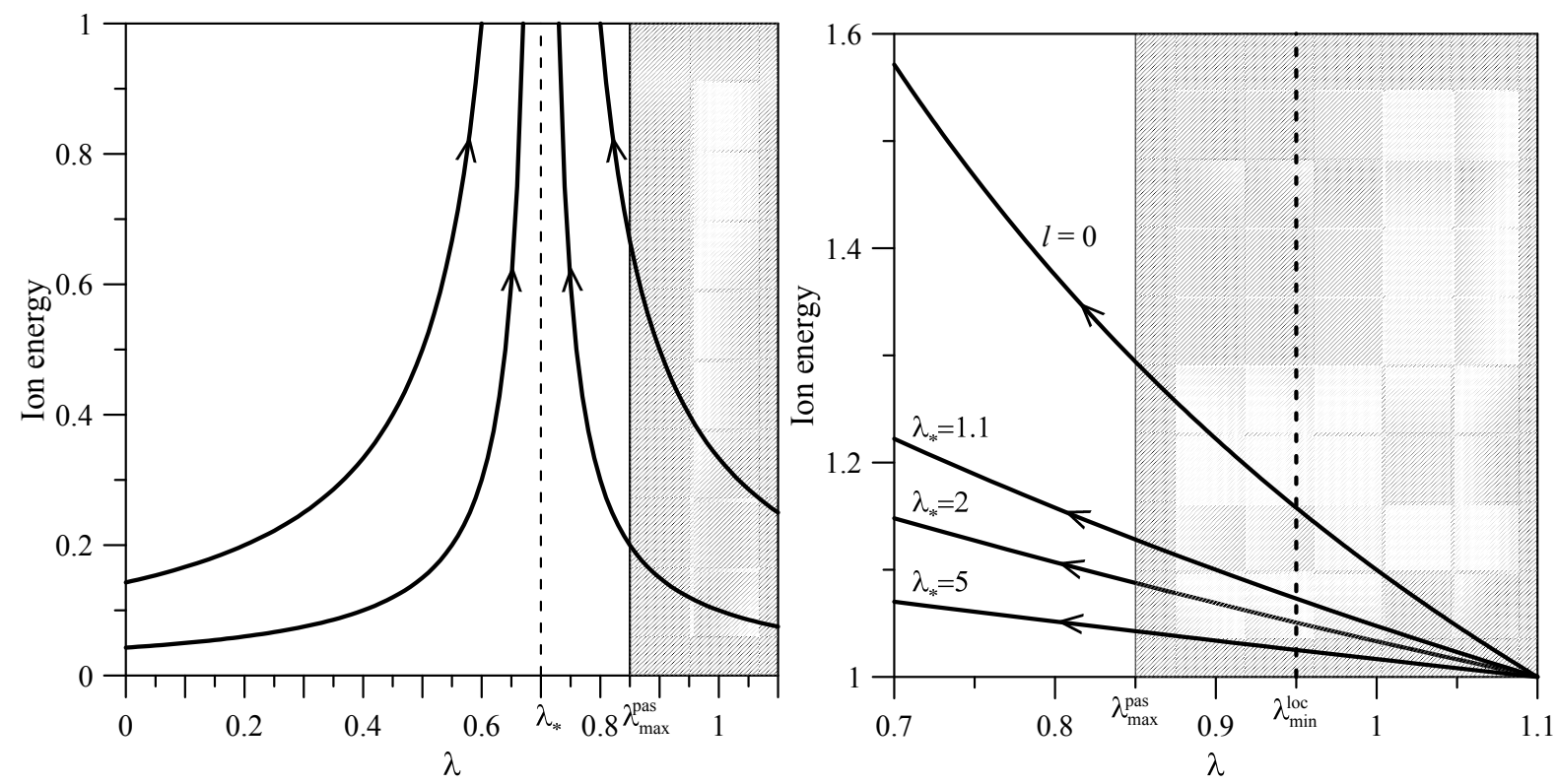

Figure 1. Curves along which quasilinear evolution of the particle distribution function takes place in the $(\lambda, \mathcal{E})$ plane: Left panel, $l=1, \lambda_{*}=0.7$; Right panel, $l=-1$ and various $\lambda_{*}$ (three lower curves) and $l=0$ (upper curve). The arrows show the direction of the evolution during RF heating. The region of trapped particles is shadowed. The ion energy is measured in arbitrary units; $\lambda_{\text {max }}^{\text {pas }}=0.85$ and $\lambda_{\text {min }}^{l o c}=0.95$ are the maximum $\lambda$ of passing particles and the minimum $\lambda$ of locally trapped ones at half-plasma radius in $\mathrm{W} 7-\mathrm{X}$ in the high mirror configuration, see figure $\mathrm{A} 2$.

an orbit transformation of deeply trapped particles to passing ones when $l<0$. When $l>0$, only marginally trapped particles (transitioning ones in the locally passing state) can be transformed to passing ones if $\Delta \mathcal{E} \sim 10 \%$, and a much larger increase of the particle energy is required for orbit transformations of deeply trapped particles. Only this case $(l>0)$, as will be shown later, may be of interest for W7-X where the RF antenna is designed for the ICRH heating.

In contrast, the acceleration of passing ions leads to an increase of $\lambda$, which eventually causes them to cross the separatrix between the passing and trapped regions when $\lambda_{*}>\lambda_{\text {max }}^{\text {pas }} / l$ if $l>0$. In this case the wave-particle interaction produces trapped ions and decreases the population of passing ones.

The transformation of trapped energetic ions to passing ones can also take place as a result of plasma instabilities driven by the energetic ions. For this to occur, the $l>0$ resonance involving both trapped and passing energetic ions should be responsible for the instability, but $\lambda_{*}$ should exceed $\lambda_{\text {max }}^{\text {pas }}$, as shown in figure 2 .

In this analysis, the inhomogeneity of the magnetic field was neglected, but this restriction can be removed by simply replacing $B_{0}$ in $\omega_{B i}$ and $\lambda$ by its average value on the magnetic axis, $\bar{B}$. The equations (5), (6) then only contain constants of the particle motion. Therefore, the pictures shown in figures 1 and 2 are valid not only for times much shorter than the bounce time $\tau_{b}$ (when the use of the constant-magneticfield-approximation is justified) but also for times exceeding $\tau_{b}$ and realistic magnetic 




Figure 2. Same as figure 1 (but for a different range of $\lambda$ ) in the case when quasilinear evolution is associated with an instability (rather than with RF injection) driven through the $l=1$ resonance. The energy of the resonant particles then drops, as indicated by arrows.

configurations. This is not surprising since the ratio $\lambda / \lambda_{*}$ does not depend on the magnetic field,

$$
\frac{\lambda}{\lambda_{*}} \propto \frac{M}{e} \frac{\mu_{p}}{\mathcal{E}} \omega
$$

where $M$ and $e$ are the particle mass and electric charge, respectively. It is merely a matter of convenience what magnitude of the magnetic field to use in the definition of $\lambda$ and $\lambda_{*}$. We shall use $B_{0}=\bar{B}$.

\subsection{Wave polarization}

The $l$-th term in the quasilinear diffusion coefficient is related to the wave field as follows:

$$
D_{l} \propto\left|e^{-i \psi} E_{+} J_{l-1}(\xi)+e^{i \psi} E_{-} J_{l+1}(\xi)\right|^{2},
$$

where $E_{ \pm}=0.5\left(\tilde{E}_{r} \pm i \tilde{E}_{b}\right), \quad \tilde{E}_{r}$ and $\tilde{E}_{b}$ are components of the wave electric field, the subscripts $r$ and $b$ refer to the radial and bi-normal directions, respectively, $\psi$ is defined by $k_{r}=k_{\perp} \cos \psi, k_{b}=k_{\perp} \sin \psi, J_{l}(\xi)$ is a Bessel function of the $l$-th order, and $\xi=k_{\perp} \rho_{\perp}$. When $\xi \ll 1$ (which is typically the case), the first term in equation (8) mainly contributes for $l=1$, whereas the second term dominates for $l=-1$. This 
implies that the $E_{+}$-component of the electric field is mainly responsible for energy transfer to the particles at the $l=1$ resonance, whereas the $E_{-}$-component dominates if $l=-1$.

The wave polarization is therefore of importance, and is determined by the bulk plasma when the number of fast ions is small. In the approximation of a homogeneous plasma it can be obtained from the Maxwell wave equations $\mathbf{k} \times \tilde{\mathbf{B}}=-(\omega / c) \hat{\epsilon} \tilde{\mathbf{E}}$ and $\mathbf{k} \times \tilde{\mathbf{E}}=(\omega / c) \tilde{\mathbf{B}}$, where a tilde labels perturbations, $\mathbf{k}$ is the wave vector, and $\hat{\epsilon} \equiv \epsilon_{i j}$ the dielectric tensor. For waves with $\tilde{E}_{\|}=0$ (ideal MHD waves) and $k_{b}=0$, these equations are reduced to

$$
\begin{aligned}
& \left(\epsilon_{1}-N_{\|}^{2}\right) \tilde{E}_{r}+i \epsilon_{2} \tilde{E}_{b}=0, \\
& -i \epsilon_{2} \tilde{E}_{r}+\left(\epsilon_{1}-N^{2}\right) \tilde{E}_{b}=0,
\end{aligned}
$$

where $N=c k / \omega$ is the refractive index, $N_{\|}=c k_{\|} / \omega, k_{\|}$is the longitudinal wavenumber, $\epsilon_{1} \equiv \epsilon_{r r}$, and $\epsilon_{2}=-i \epsilon_{r b}$. Here, an orthogonal frame $\left(\mathbf{e}_{r}, \mathbf{e}_{b}, \mathbf{e}_{\|}\right)$is used, with $\mathbf{e}_{\|}=\mathbf{B}_{0} / B_{0}$. The permeability tensor components in (9), (10) are well known. In the cold plasma approximation [i.e., when $\left(\omega-l \omega_{B i}\right)^{2} \gg k_{\|}^{2} v_{T i}^{2},\left(\omega-l \omega_{B e}\right)^{2} \gg k_{\|}^{2} v_{T e}^{2}$, and $k_{\perp} \rho_{i} \ll 1$ ] for waves with frequencies $\omega \ll \min \left(\omega_{p i}, \sqrt{M_{e} / M_{i}} \omega_{B e}\right)$ they become [8]

$$
\epsilon_{1}=\sum_{i} \frac{\omega_{p i}^{2}}{\omega_{B i}^{2}-\omega^{2}}, \quad \epsilon_{2}=\sum_{i} \frac{\omega}{\omega_{B i}} \frac{\omega_{p i}^{2}}{\omega_{B i}^{2}-\omega^{2}},
$$

where $\omega_{p i}$ denotes the plasma frequency of species $i$. The expressions (11) are valid independently of the direction of the wave vector component perpendicular to the magnetic field. Therefore, our assumption $k_{b}=0$ does not affect the properties of the waves although it does simplify the equations.

We obtain from (9)-(11) the familiar result that Alfvén waves (AW) and fast magnetoacoustic waves (FMW) with $\omega^{2} \ll \omega_{B i}^{2}$ are linearly polarized, see, e.g. [8]. To see this, we note that AWs $\left(\omega=k_{\|} v_{A}\right)$ are described by the equation $\epsilon_{1}=N_{\|}^{2}$ and, therefore, it follows from equation (9) that $\tilde{E}_{b}=0$. Fast magnetoacoustic waves with $\omega=k v_{A}$ satisfy the equation $\epsilon_{1}=N^{2}$, which leads to $\tilde{E}_{r}=0$. In both cases $\left|E_{+}\right|=\left|E_{-}\right|$.

On the other hand, when the wave frequency approaches the ion gyrofrequency of one of the ion species, $\omega=\omega_{B i}, \epsilon_{1}=\epsilon_{2} \rightarrow \infty$, then $N_{\|}^{2}$ is negligible, and equations (9), (10) are reduced to $\tilde{E}_{r}+i \tilde{E}_{b}=0$, so that $E_{+}=0$ and $E_{-} \neq 0$. Note that this is a reason why the $l>1$ resonances are to be used (and, hence, why effects of finite Larmor radius are important) for ICRF heating of a plasma with a single ion species. (However, strictly speaking, the cold-plasma-approximation does not work when $\omega=\omega_{B i}$.) When the wave frequency is close to $\omega_{B i}, E_{+} \neq 0$ but is small, see equation (40) and analysis in subsection 3.2 .

In the limit $\omega^{2} \gg \omega_{B i}^{2}$ equation (9) yields $\tilde{E}_{b} / \tilde{E}_{r}=i \omega_{B i} / \omega$ for FMWs $\left(\omega=k_{\perp} v_{A}\right)$ when $N_{\|}^{2} \ll \epsilon_{1}$, whereas $\tilde{E}_{b} / \tilde{E}_{r}=-i k_{\|} / k_{\perp}$ for helicons $\left(\omega=k_{\|} k_{\perp} v_{A}^{2} / \omega_{B i}\right)$ when $N_{\|}^{2} \gg \epsilon_{1}$ and $k_{\|}^{2} \ll k_{\perp}^{2}$. 
The same results can be obtained from the following relation, which follows from equation (9), [8]:

$$
\frac{E_{+}}{E_{-}}=-\frac{\epsilon_{-}-N_{\|}^{2}}{\epsilon_{+}-N_{\|}^{2}},
$$

where $\epsilon_{ \pm}=\epsilon_{1} \pm \epsilon_{2}$ with

$$
\begin{aligned}
& \epsilon_{+}=-\sum_{i} \frac{\omega_{p i}^{2}}{\omega_{B i}\left(\omega-\omega_{B i}\right)}, \\
& \epsilon_{-}=\sum_{i} \frac{\omega_{p i}^{2}}{\omega_{B i}\left(\omega+\omega_{B i}\right)} .
\end{aligned}
$$

\subsection{Resonances}

2.3.1. Local and global resonances. In the quasilinear approximation, only particles interacting resonantly with the waves can undergo orbit transformations caused by an RF field or plasma instabilities.

There are two kinds of resonances: local and global, see, e.g., [11]. Local resonances do not depend on the particle orbits and are therefore similar in tokamaks and stellarators. Global resonances contain orbit-averaged frequencies of the particle motion, and are effective on time scales exceeding that of the motion of particle guiding centers.

Let us introduce a characteristic time of the resonance interaction, $\tau^{r e s}$, which will determine the time of the quasilinear evolution of the distribution function. This time should be small enough in order to prevent energetic ions from escaping to the wall:

$$
\tau^{\text {res }}<\tau_{n},
$$

where $\tau_{n}$ is the lifetime of escaping fast ions. The time $\tau^{r e s}$ is much longer than the wave period. Therefore, we can write $\tau^{r e s}=Q / f_{w}$, where $f_{w}$ is the wave frequency in $\mathrm{Hz}$ and $Q=\omega \tau^{\text {res }} /(2 \pi) \gg 1$. On the other hand, $\tau_{n} \sim \tau_{s b}$, where $\tau_{s b}$ is the period of superbanana motion for locally trapped particles with orbits intersecting the wall. For these particles equation (15) reduces to

$$
f_{w}>\frac{Q}{\tau_{s b}}
$$

In particular, in W7-X $\tau_{s b} \sim 1 \mathrm{~ms}$ (see Appendix A) and equation (16) reads: $f_{w}>Q$ $\mathrm{kHz}$. For instance, when $Q \leq 10^{3}$, this condition can be satisfied even for low frequency waves, $\omega \ll \omega_{B i}$. Moreover, equation (16) should be replaced with a less restrictive condition for transitioning particles whose confinement time exceeds $\tau_{s b}$.

Typically $\tau_{s b}$ is much longer than the particle bounce or transit time $\tau_{b}$. For times exceeding $\tau_{b}$ the energy exchange between particles and waves in linear and quasilinear theories can be described by means of relations that contains the integral [9]

$$
\int_{0}^{\tau_{b}} e^{i \psi(\tau)} d \tau
$$


where

$$
\psi(\tau)=\int_{0}^{\tau}\left[\omega-l \omega_{B}\left(\tau_{1}\right)-m \dot{\vartheta}+n \dot{\varphi}\right] d \tau_{1} .
$$

Dots here denote $\tau_{1}$-derivatives, $\vartheta$ and $\varphi$ the poloidal and toroidal angles, $m$ and $n$ are the corresponding mode numbers, and all perturbations are chosen to be of the form $\tilde{X} \propto \exp (-i \omega t+i m \vartheta-i n \varphi)$. When

$$
\omega \gg \omega_{b} \equiv \frac{2 \pi}{\tau_{b}},
$$

most of the contribution to the integral (17) comes from the point $\tau_{*}$ of stationary phase, determined by $d \psi / d \tau_{*}=0$, which represents the local resonance given by

$$
\Omega_{l} \equiv \omega-l \omega_{B i}-k_{\|} v_{\|}^{r e s}=0,
$$

where $v_{\|}$is the particle velocity along the magnetic field, $k_{\|}=(m \iota-n) / R$, and we have approximated $\dot{\vartheta}=\iota \dot{\varphi}=\iota v_{\|} / R$. In particular, $\omega_{b}=6.6 \times 10^{5} \mathrm{~s}^{-1}$ for locally trapped $55-\mathrm{keV}$ protons in W7-X, see section 3. This implies that local resonances exist in this case for wave frequencies exceeding $100 \mathrm{kHz}$. Waves with $\omega \sim \omega_{B i}$ satisfy this condition, whereas for waves with frequencies comparable to $\omega_{b}$ only global resonances are meaningful. Both local and global resonances exist for $\omega_{b} \ll \omega \lesssim \omega_{B i}$.

Global resonances for passing particles are given by

$$
\omega-l\left\langle\omega_{B i}\right\rangle-\left\langle k_{m+\mu, n+\nu N} v_{\|}\right\rangle=0,
$$

where $\langle(\ldots)\rangle$ denotes bounce/transit-time averaging, $k_{m+\mu, n+\nu N}=[(m+\mu) \iota-(n+$ $\left.\left.\nu N_{f}\right)\right] / R$, where $\mu$ and $\nu$ are integers that determine the Fourier components of the equilibrium magnetic field, $N_{f}$ is the number of field periods [13]. Equation (21) is valid for particles with narrow orbit widths $\left(\Delta_{r}\right)$. Finite $\Delta_{r}$ leads to additional resonances [14]. When $k_{\|} R / \iota \gg 1$ and $\mu \sim 1, \nu=0$, this equation determines $\left\langle v_{\|}\right\rangle^{\text {res }}$, which approximately coincides with $v_{\|}^{\text {res }}$ determined by the local resonance. In general, however, this is not the case.

Global resonances for trapped particles are determined by

$$
\omega-l\left\langle\omega_{B i}\right\rangle-s \omega_{b}=0
$$

where $s$ is an integer and the precession frequency is neglected. This equation is true both for particles oscillating within toroidal angles $\Delta \varphi=2 \pi / N_{f}$ (locally trapped particles and transitioning particles in the trapped state) and for particles oscillating within $\Delta \varphi \sim 1 / \iota$ (transitioning particles in the locally passing state). The magnitudes of $\omega_{b}$ are different for these two groups of trapped particles: $\omega_{b}^{l o c} \sim \iota^{-1} N_{f} \omega_{b}^{l / p}$, where the superscripts $l o c$ and $l / p$ label bounce frequencies in the locally trapped and locally passing states, respectively.

Note that orbits of transitioning particles in the locally passing state mainly lie in a region close to the outer midplane of the torus, see figure A1 in Appendix A. Therefore, it is necessary that the wave field be present in this region to influence locally passing particles. 
2.3.2. Analysis of local resonances. For given values of $|l|, \omega$ and $k_{\|}$, the diffusion coefficient is $\sum_{l,-l} D_{l}=D_{|l|}\left(v_{\|}^{l>0}\right)+D_{-|l|}\left(v_{\|}^{l<0}\right)$. In the approximation $\xi \ll 1$, the $E_{+_{-}^{-}}$ term mainly contributes for $l>0$, whereas the $E_{-}$-term dominates for $l<0$ :

$$
\sum_{l} D_{l} \propto\left[\left|E_{+}\right|^{2} \delta\left(\Omega_{l>0}\right)+\left|E_{-}\right|^{2} \delta\left(\Omega_{l<0}\right)\right] J_{|l|-1}^{2},
$$

where $l \neq 0$ and terms proportional to $J_{|l|+1}^{2}$ have been neglected. For $l=0$ we have

$$
D_{l=0} \propto\left|E_{+} e^{-i \psi}-E_{-} e^{i \psi}\right|^{2} \delta\left(\Omega_{l=0}\right) J_{1}^{2} .
$$

The radial dependence of $k_{\|}$in $\Omega_{l}$ can be neglected when $m \ll n$ or $\iota(r) \approx$ const. Assuming that this is the case (W7-X has a very small global magnetic shear) and that the field modulation $\left(\delta B_{0} / B_{0}\right)$ is small, we take $k_{\|}=$const and replace $\omega_{B i}$ by $\bar{\omega}_{B i}$ by $\omega_{B i}(\bar{B})$ in equation $(20)$, where $\bar{B}$ is the average equilibrium magnetic field on the magnetic axis. This approximation can be useful even when effects of the spatial variation of $k_{\|}$and $\omega_{B i}$ are not negligible, because it makes it possible to evaluate the longitudinal wavenumbers required to satisfy the resonance condition for a wide range of wave frequencies.

We thus choose a $k_{\|}$that satisfies $(20)$ with the gyrofrequency $\bar{\omega}_{B i}$ for beam ions having the longitudinal velocity $v_{\|}^{b}$ :

$$
k_{\|}^{+}=(\tilde{\omega}-|l|) \frac{\bar{\omega}_{B i}}{v_{\|}^{b}}, \quad k_{\|}^{-}=(\tilde{\omega}+|l|) \frac{\bar{\omega}_{B i}}{v_{\|}^{b}}, \quad k_{\|}^{0}=\tilde{\omega} \frac{\bar{\omega}_{B i}}{v_{\|}^{b}},
$$

where $k_{\|}^{+} \equiv k_{\|}(l>0), k_{\|}^{-} \equiv k_{\|}(l<0), k_{\|}^{0} \equiv k_{\|}(l=0)$, and $\tilde{\omega}=\lambda_{*}{ }^{-1} \equiv \omega / \bar{\omega}_{B i}$. Various consequences of this choice are now discussed:

(i) We first consider the case where $k_{\|}^{+}$is selected and assume $\tilde{\omega}>\left(\lambda_{\text {max }}^{\text {pas }}\right)^{-1}$ in order to enable $\lambda$ to decrease for trapped particles. Then the first term in (23) involves particles with the velocity $v_{\|}^{b}$ and depends on $E_{+}$. The second term, associated with $E_{-}$, arises due to the resonance (20) with $l<0$ and involves particles with

$$
v_{\|}^{\text {res }}(l<0)=\frac{(\tilde{\omega}+|l|)}{(\tilde{\omega}-|l|)} v_{\|}^{b} .
$$

These particles can be transformed into passing ones. However, equation (26) is satisfied only for $v_{\|}^{\text {res }} \gg v_{\|}^{b}$, unless $\tilde{\omega} \gg l$ (for given $l$ ).

(ii) The second case is that when $k_{\|}^{-}$selected, and requires the smallest energy to increase the particle pitch (to decrease $\lambda$ ), especially when $\omega \ll \omega_{B i}$, see subsection 2.1. In addition, $E_{-}^{2}$ may well exceed $E_{+}^{2}$ and, therefore, most the RF energy can be transferred to fast ions with $v_{\|}=v_{\|}^{b}$ because the term in (23) involving particles with $v_{\|}^{b}$ is determined by $E_{-}$. The term, associated with $E_{+}$, affects particles with

$$
v_{\|}^{\text {res }}(l>0)=\frac{(\tilde{\omega}-|l|)}{(\tilde{\omega}+|l|)} v_{\|}^{b}
$$

This term arises due to the $l>0$ resonance, and therefore its effect is desirable (decreasing $\lambda$ ) only when the wave frequency is sufficiently high, $\tilde{\omega}>\lambda^{-1} \sim 1$. When $\tilde{\omega} \lesssim l$, the resonant particles determined by $(27)$ are absent and, hence, the $l>0$ 
resonance does not work. For instance, for $\tilde{\omega}=0.7, v_{\|}^{\text {res }}(l=1)=-0.18 v_{\|}^{b}$. However, when the wave frequency is very low, $\omega \ll \omega_{B i}, v_{\|}^{r e s}(l>0) \approx-v_{\|}^{b}$. It is not clear whether such waves can provide orbit transformation of trapped particles since the sign of their longitudinal velocity changes during the bounce motion. As a result, the $l>0$ resonance may cancel the positive effect of the $l<0$ resonance. This cancellation can be prevented by effects we have neglected, such as finite orbit width, radial quasilinear displacement of the fast ions, and the inhomogeneity of the wave field.

(ii) The third case ( $\omega_{b} \ll \omega \ll \omega_{B i}$ and $k_{\|}^{0}$ selected) requires a little bit more energy for the orbit transformation than the $k_{\|}^{-}$case. On the other hand, waves with $\omega \ll \omega_{B i}$ can be used without any threat that a positive effect of the waves on one part of the orbit (where $v_{\|}>0$ ) will be cancelled on another part (where $v_{\|}<0$ ): the only resonant velocity is $v_{\|}^{b}$ (for given $k_{\|}^{0}$ ) and $d \mathcal{E} / d \lambda$ is always negative, see right panel in figure 1 . One more positive feature is that $k_{\|}^{0} \ll \rho_{\| b}^{-1}$ for $\omega \ll \omega_{B i}$.

It is clear that the proposed way of transforming trapped particles into passing ones can be of practical importance only if the waves interact with fast ions having various longitudinal velocities. This implies that waves with the corresponding spectrum of wavenumbers, not only those determined by equation (25), should be present (for a given wave frequency). The presence of a $k_{\|}$-spectrum is also required for the quasilinear evolution of the fast-ion distribution. It follows from equation (20) that the region of resonance velocities is about $\left|\Delta v_{\|} / v_{\|}\right|=\left|\Delta k_{\|} / k_{\|}\right|$, where $\Delta k_{\|}$is the effective width of the spectrum. Note that in reality the resonance region is somewhat wider because of the finite resonance width, which depends on the wave amplitude.

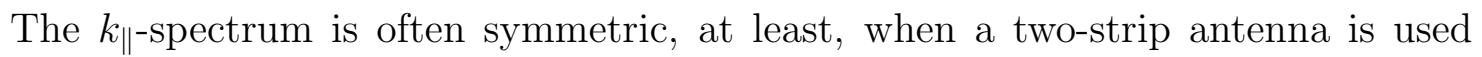
[except for the case of $(0 \pm \pi / 2)$ phasing] [15]. Due to this circumstance, waves with both $k_{\|}>0$ and $k_{\|}<0$ are generated. Trapped particles are then affected by the RF field on both legs of their bounce orbits.

The antenna $k_{\|}$-spectrum may include those $k_{\|}$which provide resonant interaction between the RF field and well-passing ions with $\lambda<\lambda_{*}$. This will lead to ion acceleration accompanied by a decrease of the pitch $\chi$ when the $l>0$ resonance is responsible for the wave-particle interaction. Nevertheless, the transformation of passing particles into the trapped ones will not occur due to the condition $\lambda_{*}<\lambda_{\text {max }}^{\text {pas }}$. If NBI heating is sufficient to sustain the desired plasma temperature, one has to exclude or minimize this acceleration of the passing ions by ICRH. This can be achieved by imposing a restriction on what values for $k_{\|}$for which the antenna power spectrum is large. This restriction is

$$
k_{\|}>k_{*} \equiv \frac{\chi_{m a x}^{\operatorname{trap}}}{\chi_{*}} k_{\|}^{+}
$$

where $\chi_{*} \approx \sqrt{1-\lambda_{*}}$ and $\chi_{\max }^{t r}$ is the maximum pitch of the trapped ions. To obtain (28) we used the resonance (20) and the definition of $k_{\|}^{+}$given by (25).

Note that when the wavenumbers are determined by equation (25), the waves do not interact with electrons through the cyclotron resonance, but the $l=0$ resonance is possible. Therefore, some RF energy can be absorbed directly by the electrons. 
2.3.3. On global resonances of trapped particles. Equation (22) for the trapped particles does not depend on $k_{\|}$, and the resonance region for given $s$ is therefore determined by the wave amplitude alone. A particle trapped in the wave field moves thus regularly in the resonance island unless it overlaps with other islands [16]. On the other hand, the bounce frequency is much smaller (by a factor of $10^{2}-10^{3}$ ) than $\omega_{B i}$ and, therefore, bounce resonances may overlap, while resonances corresponding to different cyclotron harmonics are well separated. For this to be the case the wave amplitude should be sufficiently large and the frequency should not be very close to $l \omega_{B i}$,

$$
\left|\omega-l \omega_{B i}\right| \gg \omega_{b}
$$

From this equation, $s \gg 1,\left|\Delta \omega_{b} / \omega_{b}\right|=1 / s \ll 1\left(\Delta \omega_{b}\right.$ is the distance between the neighbouring bounce resonances) and, therefore, the summation over $s$ in the equation for the diffusion coefficient can be replaced by an integration, the main contribution to the integral coming from the local resonance, see, e.g., [17]. When bounce resonances overlap, the particle motion is stochastic and equations (5), (6) with the gyrofrequency averaged over the bounce period are applicable.

For low-frequency waves, $\omega \sim \omega_{b}$ local resonances are absent. Wave-particle interaction is then realized through the global resonance $\omega=s \omega_{b}$ with $s \sim 1$.

\section{Application to Wendelstein $7-\mathrm{X}$}

\subsection{Pitches of trapped particles}

The longitudinal velocity of a particle is not a constant of the motion. For the trapped particles it varies from zero to a maximum magnitude, $\left|v_{\| \max }\right|$, implying that these particles can interact resonantly with the wave provided that $\left|v_{\|}^{\text {res }}\right|$ determined by equation (20) does not exceed $\left|v_{\| \max }\right|$ (or $\left|\chi^{\text {res }}\right| \leq \max |\chi| \equiv \chi_{\max }$ for the given particle energy). The quantity $\chi_{\max }$ is, of course, a constant of the motion and be written as

$$
\chi_{\max }=\left(1-\frac{1}{1+\alpha} \frac{B_{\min }}{\bar{B}}\right)^{1 / 2}
$$

where $B_{\text {min }}$ is the minimum magnetic field along a particle orbit, $\alpha \equiv \lambda^{-1}-1$. Equation (30) follows from the definition of $\lambda=\mathcal{E}_{\perp} \bar{B} /\left(\mathcal{E} B_{0}\right)$ [we just used $\left.\mu_{p}=\mathcal{E}_{\perp} / B_{0}(r, \vartheta)\right)$ in the relation for $\lambda$ given in subsection 2.1].

To calculate $\chi_{\max }$ we have to specify the equilibrium magnetic field, which we take to be in a form that includes the main Fourier harmonics in Wendelstein 7-X [7]:

$$
B_{0}=\bar{B}\left[1+\epsilon_{0}+\epsilon_{m} \cos \left(N_{f} \varphi\right)-\epsilon_{h} \cos \left(\vartheta-N_{f} \varphi\right)-\epsilon_{t} \cos \vartheta\right]
$$

where $\epsilon_{0}, \epsilon_{m}, \epsilon_{h}$, and $\epsilon_{t}$ are absolute values of the diamagnetic, mirror, helical, and toroidal harmonics, respectively. One sees that when $\epsilon_{m} \gg \epsilon_{h}-\epsilon_{t}$, which is true for the high-mirror configuration in W7-X, $B_{\min }$ can be evaluated as

$$
B_{\text {min }}=\bar{B}\left(1+\epsilon_{0}-\epsilon_{m}\right) \text {. }
$$




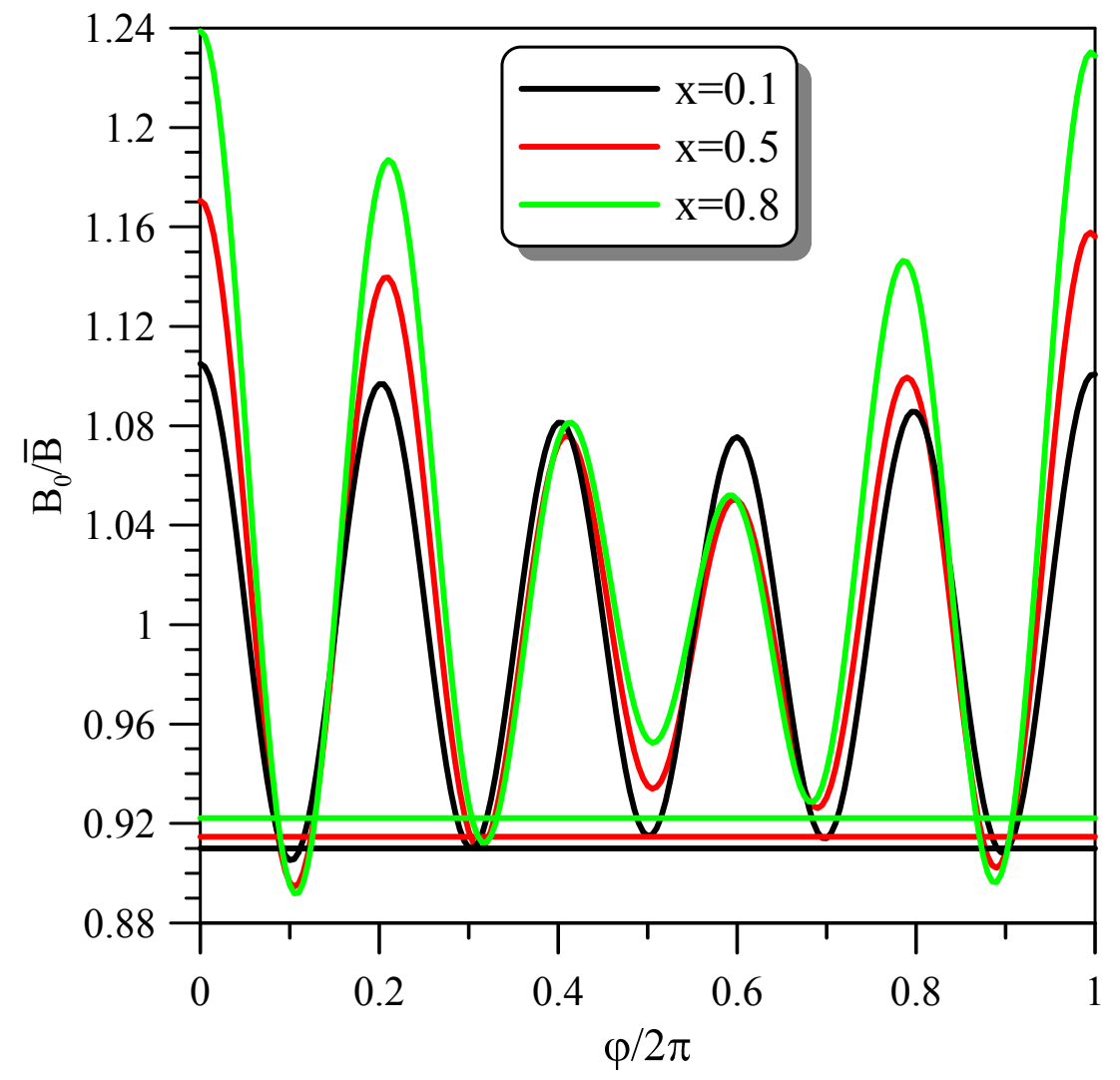

Figure 3. Variation of the magnetic field strength along the field line passing through the point $\left(\vartheta=\pi, \varphi=0\right.$ ) as described by equation (31), and $B_{\min }$ (horizontal lines) determined by equation (32) at different radii, $x=r / a, a$ is the plasma radius. Local maxima are located at $\varphi_{\max } \approx 2 \pi j / N_{f}$ and local minima at $\varphi_{\min } \approx 2 \pi(j+0.5) / N_{f}$, with $j=0,1,2, \ldots$ and $N_{f}=5 ; \vartheta=\iota \varphi-\pi$.

For illustration, the variation of the field strength along the field line $(\iota \varphi-\vartheta=$ const $)$ using equation (31) is shown figure 3. As expected, the minima of $B_{0}$ depend only weakly on $\varphi$ (especially in the plasma core region), and in addition, they do not vary much with radius and are well approximated by (32).

The pitch parameter $\alpha$ of the two groups of trapped particles - locally trapped ones (labelled by the superscript "loc") and transitioning ones (labelled by the superscript "tran") - lies in the intervals [18]:

$$
\begin{aligned}
& \alpha_{\min }<\alpha^{l o c}<\epsilon_{0}+\epsilon_{m}-\epsilon_{h}-\epsilon_{t}, \\
& \epsilon_{0}+\epsilon_{m}-\epsilon_{h}-\epsilon_{t}<\alpha^{\text {tran }}<\epsilon_{0}+\epsilon_{m}+\epsilon_{h}+\epsilon_{t},
\end{aligned}
$$

where $\alpha_{\min }$ is determined by the equation $\kappa^{2}(r, \vartheta)=0, \kappa$ is the particle trapping parameter, $\kappa^{2}(r, \vartheta)=1$ is the separatrix between the locally trapped particles and locally passing ones.

For passing particles we have

$$
\alpha^{\text {pass }}>\epsilon_{0}+\epsilon_{m}+\epsilon_{h}+\epsilon_{t} .
$$




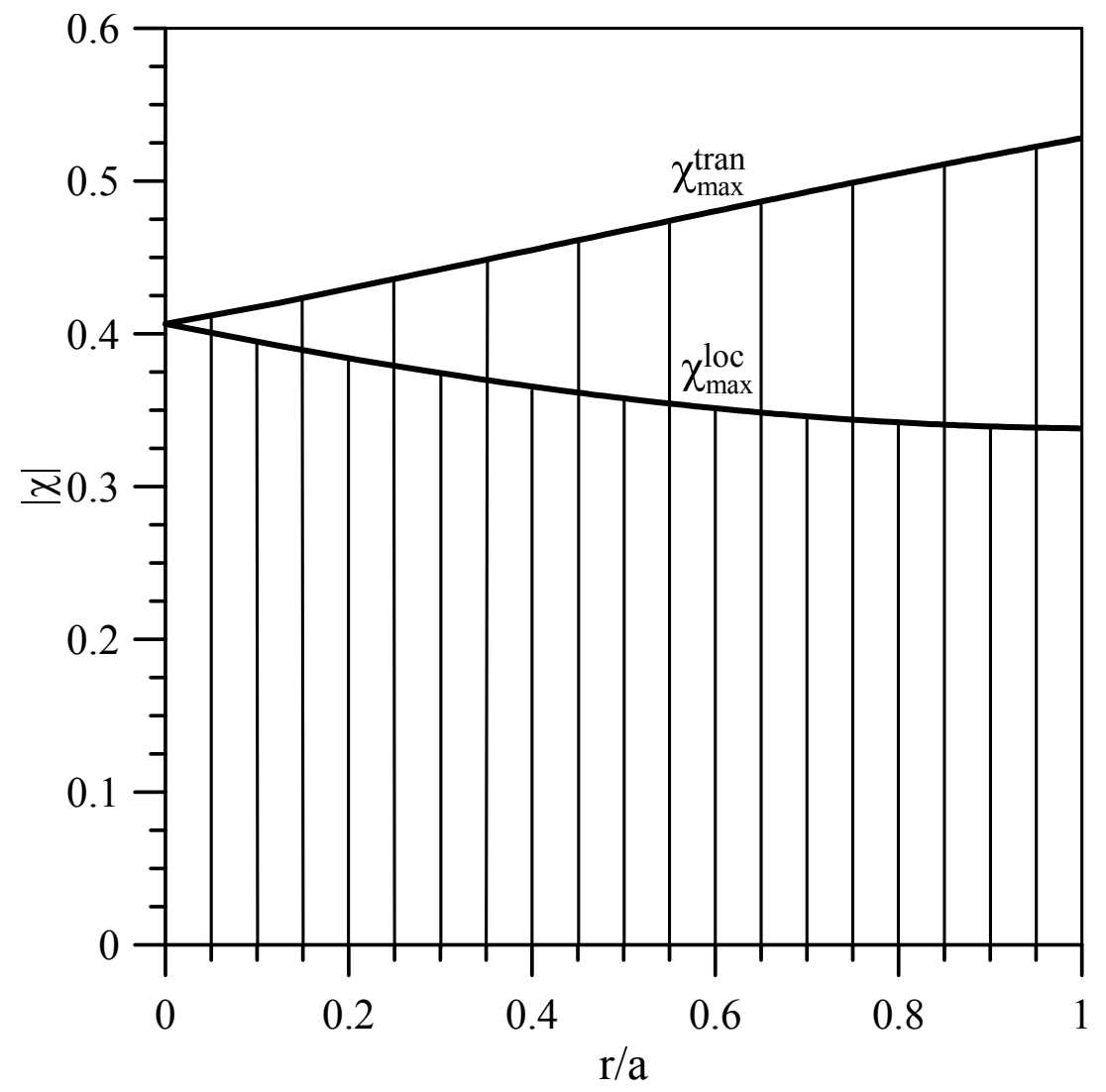

Figure 4. Pitch $\chi \equiv v_{\|} / v$ of locally trapped NBI ions and transitioning ones in W7-X. The ranges of pitches during the particle orbital motion are shown by vertical lines. The maximum values of $\chi$ are shown by bold lines.

Using (30), (32), (33) and (34) we obtain:

$$
\begin{aligned}
& \chi_{\text {max }}^{l o c}=\left(\frac{2 \epsilon_{m}-\epsilon_{h}-\epsilon_{t}}{1+\epsilon_{0}+\epsilon_{m}-\epsilon_{h}-\epsilon_{t}}\right)^{1 / 2}, \\
& \chi_{\max }^{\text {tran }}=\left(\frac{2 \epsilon_{m}+\epsilon_{h}+\epsilon_{t}}{1+\epsilon_{0}+\epsilon_{m}+\epsilon_{h}+\epsilon_{t}}\right)^{1 / 2} .
\end{aligned}
$$

In particular, $\chi_{\max }^{\text {loc }}=0.35$ and $\chi_{\max }^{\text {tran }}=0.48$ at $r / a=0.6$ in the W7-X high-mirror configuration.

\subsection{Specific examples}

3.2.1. The use of available tools. The maximum energy of NBI ions in W7-X will be $55 \mathrm{keV}$ for protons and $60 \mathrm{keV}$ for deuterons. The RF frequency lies in the range $f_{w}=25-38 \mathrm{MHz}$, and the location of the maximum in the antenna $k_{\|}$-spectrum varies from $\sim 3 \mathrm{~m}^{-1}$ to $\sim 11 \mathrm{~m}^{-1}$, depending on the phasing [15]. The high- $k_{\|}$wing of the spectrum reaches $k_{\|} \sim 20 \mathrm{~m}^{-1}$. In addition, the antenna could also be operated at the frequency $f_{w}=76 \mathrm{MHz}$ [15], but this requires internal modifications to some capacitors. 
It follows from equations (25) that the resonance condition can be consistent with the W7-X antenna spectrum only for $l>0$. Otherwise, the required wavenumber is too large, $k_{\|}>(\rho \chi)^{-1}>100 m^{-1}$, where $\rho=v^{b} / \bar{\omega}_{B i}$ is the fast ion Larmor radius. In this case the trapped particles can be converted to passing ones provided that

$$
\tilde{\omega}>l\left(\lambda_{\text {max }}^{\text {pas }}\right)^{-1} \text {. }
$$

This condition is generic, it does not depend on the magnetic field and the wavenumbers, and it guarantees that the reverse process - the transformation of passing ions to trapped ones - will not occur. Taking $\lambda_{\text {max }}^{\text {pas }}=0.83$ (we rely on figure A2) we conclude that the required frequency is $\tilde{\omega} \gtrsim 1.2 l$.

However, the maximum frequency of the lower-frequency band (38 $\mathrm{MHz})$ corresponds to $\tilde{\omega}=1.1$ (for protons) for the reference magnetic field $\bar{B}=2.27 \mathrm{~T}$ adopted for this magnetic configuration. To provide $\tilde{\omega}=1.2$, the magnetic field should be decreased by a factor of 1.09 to $\bar{B}=2.08 \mathrm{~T}$. (Increasing the frequency by a similar factor, to $41.5 \mathrm{MHz}$, is not possible.) The RF field will then act to increase the pitch $\chi$ of any injected protons. (Only relatively few discharges with deuterium injection will be performed in the first NBI experiments.)

In contrast to (38), the resonance condition (20) and the wave polarization (12) are rather sensitive to the variation of the magnetic field within the plasma volume, since the selected value of $\bar{\omega}$ is relatively close to unity. Taking into account the inhomogeneity of the magnetic field, we can write:

$$
\begin{aligned}
& k_{\|}^{+}=\frac{\tilde{\omega}_{\alpha}-|l| b}{\rho \chi}, \\
& \frac{E_{+}}{E_{-}}=-\frac{\tilde{\omega}_{p}-b}{\tilde{\omega}_{p}+b} g,
\end{aligned}
$$

where

$$
g=\frac{1-\frac{k_{\|}^{2} \bar{v}_{A 0}^{2}}{\bar{\omega}_{B i}^{2}} \frac{b\left(\tilde{\omega}_{p}+b\right)}{1+\frac{k_{\|}^{2} v_{A 0}^{2}}{\bar{\omega}_{B i}^{2} \eta(r)}} \frac{b\left(\tilde{\omega}_{p}-b\right)}{\tilde{\omega}_{p}^{2} \eta(r)}}{.}
$$

$b=B_{0}(r, \vartheta, \varphi) / \bar{B}$, the ion density profile is given by $n_{i}=n_{i 0} \eta(r)$, and the subscripts $\alpha$ and $p$ label $\tilde{\omega}$ for the beam ions and plasma, respectively. It is clear from equation (39) that when $k_{\|} \rho \chi \ll 1$ (the case of interest for W7-X), the resonance can take place only in points with $b>1$. On the other hand, it follows from figure 3 that $b<1$ in the minima of the magnetic field. This implies that the resonance condition cannot be satisfied by deeply trapped localized particles. Such particles will not be produced in any great numbers by the NBI, see figure A2, but could result from collisional scattering of the injected ions. In the first planned NBI experiments on W7-X, only passing particles and particles with $\lambda$ in the transitioning region will be produced upon ionization. Moreover, the neoclassical radial electric field prevents orbit losses of most transitioning particles in the locally trapped state, as shown in Appendix A. Thanks to this circumstance, in order to improve the confinement of transitioning particles it is sufficient to affect them 


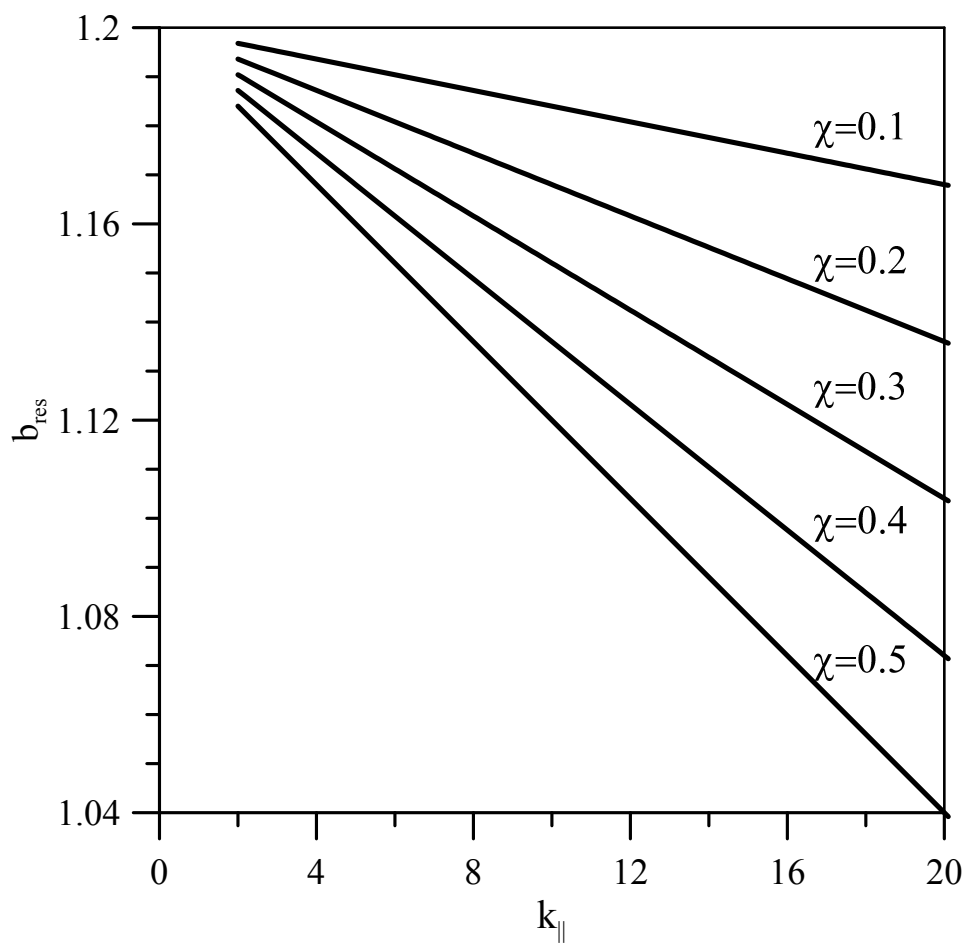

Figure 5. The resonance magnetic field $b_{\text {res }} \equiv B_{0}^{\text {res }} / \bar{B}$ determined by equation (39) with $l=1$ for the $55 \mathrm{keV}$ protons. The range $2 \mathrm{~m}^{-1}<k_{\|}<20 \mathrm{~m}^{-1}$ is relevant to the W7-X antenna spectrum, and $0<\chi<0.55$ for trapped particles and marginally passing ones. The obtained magnitudes of $b_{\text {res }}$ exist in the magnetic configuration, as seen from figure 3 and figure 6 .

by the RF field when they are in the locally passing state. The orbits of these particles are located above the local maxima shown in figure 3, namely, at $1.08<b<1.24$. This is consistent with the magnitudes of the magnetic field $1.04<b_{\text {res }}<1.2$ predicted by the resonance equation (39), see figure 5.

One more requirement for resonance with transitioning particles in the locally passing state is that it should be possible at $\vartheta \lesssim 1$, where these particles undergo bounce motion (see figure A1 for the injected with $\lambda=0.9$ ). As follows from figure 6 , this condition is fulfilled and the resonance occurs at $N_{f} \varphi \approx 2 \pi j$. The region of the 

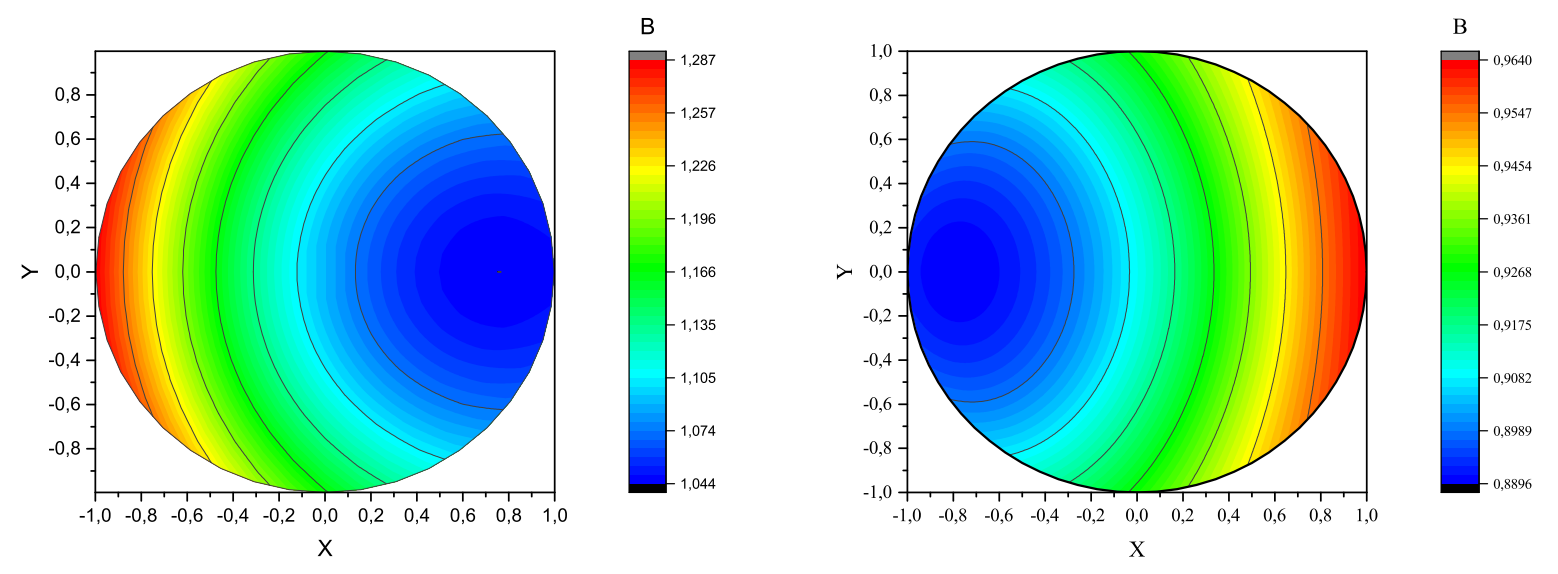

Figure 6. Contour plots (in polar Boozer coordinates) of $b \equiv B_{0} / \bar{B}$ in the highmirror configuration of W7-X in the cross sections $N_{f} \varphi=2 \pi j$ (left panel) and $N_{f} \varphi=2 \pi(0.5+j)$ (right panel), with $j=0,1,2, \ldots$ The coordinates are defined by $(x, y)=(r / a)(\cos \vartheta, \sin \vartheta)$.

locally passing state expands as $\lambda$ decreases (this follows from figure 7 ), which increases the number of resonance points on the orbits of these particles.

Finally we discuss the issue of wave polarization. When the plasma ions are of the same species as the beam ions, $\tilde{\omega}_{b}=\tilde{\omega}_{p}$. Therefore the $l=1$ resonance cannot effectively be employed in a hydrogen plasma with NBI protons because $E_{+}$is small, but in deuterium plasmas $\left(\tilde{\omega}_{p}=2.4\right)$ the polarization can be acceptable. The results of calculations shown in figure 8 confirm this. In particular, the ratio $E_{+} / E_{-} \sim 0.3$ for $k_{\|} \sim 10 \mathrm{~m}^{-1}$.

3.2.2. Other possibilities. The smallest energy for orbit transformation is required in the scenario with the $l<0$ dominant resonance, as seen from figure 1 . In this case, however, the wavenumber determined by the local resonance $\left[k_{\|}^{-}\right.$in equation (25)] is very large, $k_{\|}^{-} \gtrsim \rho_{\| b}^{-1}$ at $\omega \gtrsim \omega_{B i}$, where $\rho_{\| b}=v_{\|}^{b} / \omega_{B i}$ is the parallel Larmor radius. This scenario is thus not achievable with conventional ICRH antennas although the required frequency of the RF field is available.

Much smaller wavenumbers $\left(k_{\|}^{0} \ll \rho_{\| b}^{-1}\right)$ are required when the $l=0$ resonance dominates and $\omega \ll \omega_{B i}$. More energy is then be consumed for orbit transformation than for negative $l$, but less than in the case of $l>0$ resonance. For the local resonance to exist, the wave frequency should well exceed $\omega_{b}$. If, for example, $\omega=10 \omega_{b}^{\text {loc }}$ we obtain $k_{\|}^{0}=2 / \chi \mathrm{m}^{-2}$ for $55-\mathrm{keV}$ protons. This wave number corresponds to the avaialble antennas, but the required frequency is only $f_{w}=1 \mathrm{MHz}$.

When the condition of local resonance is not satisfied, the interaction of waves with trapped particles occurs due to the resonance $\omega=s \omega_{b}$, with $s \sim 1$, everywhere in the region where the wave field is present. In this connection, isomon modes - which are modes of Alfvénic character affected by plasma compressibility and having $m=n$ may 


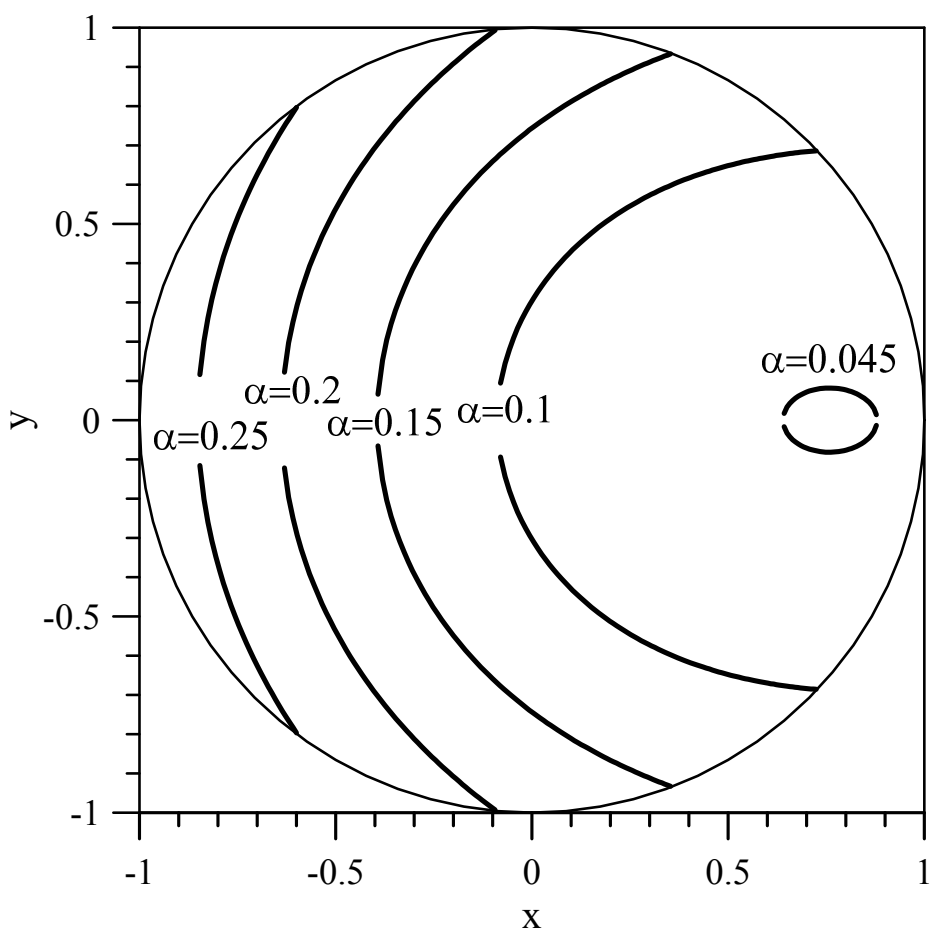

Figure 7. The $\kappa^{2}=1$ lines in W7-X high-mirror configuration for various magnitudes of the pitch parameter $\alpha$ in the absence of a radial electric field $\left(E_{0 r}=0\right)$. We observe that the region where transitioning particles are in the locally passing state (to the right of the curves) increases when $\alpha$ increases and occupies almost all the plasma cross section for $\alpha=0.25$. On the other hand, it is detached from the plasma edge at $\alpha=0.045$, which implies that collisionless diffusion [7] does not lead to losses of particles with small $\alpha$. 


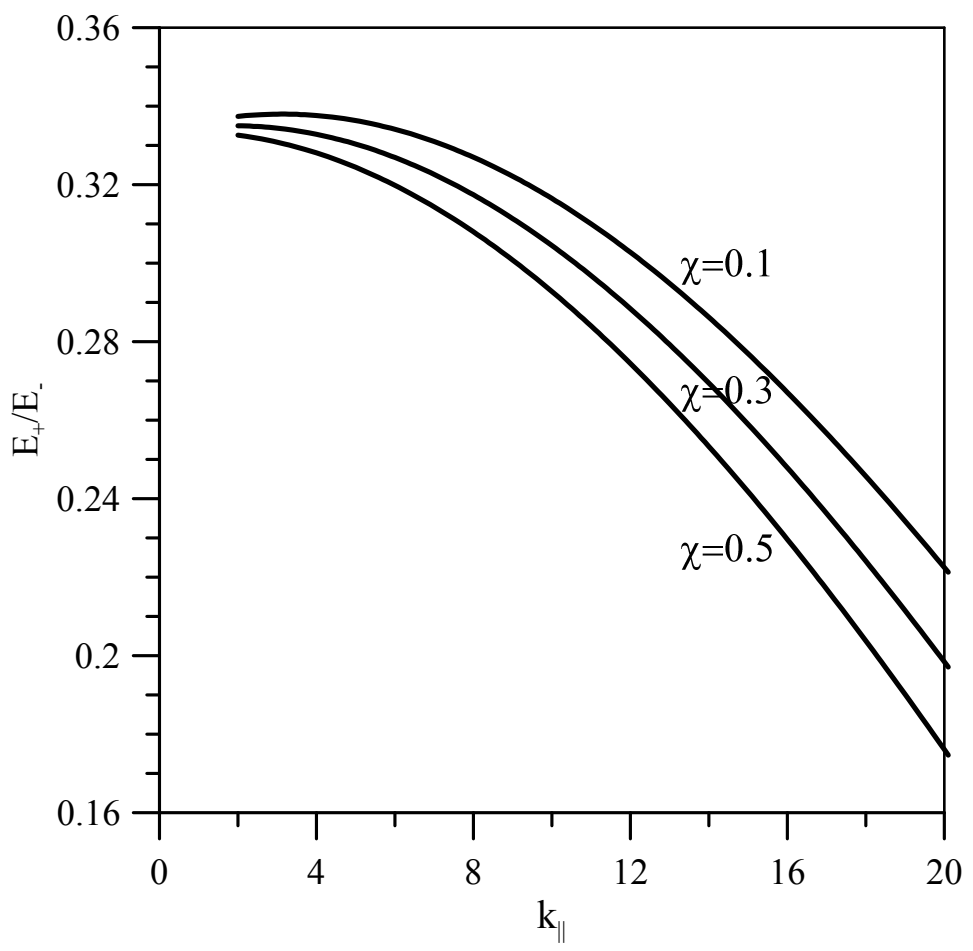

Figure 8. The ratio $E_{+} / E_{-}$for the same ranges of parallel wavenumbers and particle pitches as in figure 5. The central electron density is $n_{e}(0)=8 \times 10^{13} \mathrm{~cm}^{-3}$ and $\eta=0.9$.

be relevant. These modes are predicted to exist in W7-X due to small magnetic shear and $\iota$ close to unity [19]. When $m=n \gtrsim 1$, they occupy a considerable part of the plasma cross section and can interact resonantly with both trapped and passing NBI ions in W7-X. However, it is not clear how these modes could be excited by antennas. Note that the modes with $m=n \sim 1$ were recently observed in a low-shear region in the KSTAR tokamak [20]. In this experiment, the $m=n=3$ mode was destabilized with a current blip induced by electron cyclotron heating (ECH), which successively evolved to $m / n=2 / 2$ and $m / n=1 / 1$ modes in the core of sawtoothing discharges. 


\section{Summary and conclusions}

We have considered the possibility of improving energetic-ion confinement in stellarators by using plasma waves to modify the distribution function. The waves are assumed to result from the injection of $\mathrm{RF}$ power, but could also arise due to plasma instabilities. In either case, the aim would be to convert trapped orbits into passing ones, and a number of requirements on the wave spectrum have been identified.

In particular, it is shown that an RF field with frequency around the ion gyrofrequency of beam ions can in principle result in an increase of $\chi$ (when beam ions and plasma ions are of different species), although ICRH in current experiments on tokamaks usually leads to smaller $\chi$ and the production of trapped particles. In order to avoid the latter, the inequality $\omega>l \bar{\omega}_{B b} / \lambda_{\text {max }}^{\text {pas }}$ should be satisfied. This inequality also represents a necessary condition for the transformation of trapped orbits to passing ones. Note that this condition is generic, valid for both stellarators and tokamaks.

To satisfy it in the W7-X high-mirror configuration with the available ICRH antenna, the equilibrium magnetic field must be decreased somewhat (by nine percent) below its standard value. (This could make plasma heating with ECRH difficult since the W7-X gyrotrons operate at a fixed frequency tuned to twice the electron cyclotron frequency.) ICRH at the frequency $38 \mathrm{MHz}$ and wavenumbers in the range $2 \mathrm{~m}^{-1}<k_{\|}<20 \mathrm{~m}^{-1}$ ) is consistent with the requirement to satisfy the resonance condition for $55 \mathrm{keV}$ protons with pitches corresponding to transitioning particles, and such particles will indeed be produced in great numbers by the NBI. However, they will only interact efficiently with the ICRH wave field if the main plasma species is different from hydrogen, because of the issue with polarization. This severely limits the applicability of the scheme to W7-X. Indeed, it should be emphasized that the present study merely investigates whether wave-induced fast-ion confinement is possible in principle. Whether it will be possible in practice on $\mathrm{W} 7-\mathrm{X}$ or any other device is unclear and requires a more detailed analysis.

In principle, the use of low-frequency waves $\left(\omega \ll \omega_{B i}\right)$, for which the main resonance has $l=0$, is more attractive since less energy is required to convert trapped particles into passing ones. In addition, in this case the decrease of pitches of well passing particles is intrinsically absent. However, it is neither clear what plasma waves could be used for this purpose, nor how they would be launched.

A by-product of our analysis is that the neoclassical electric field changes the confinement time and pitch-angle spectrum of the lost NBI ions in W7-X. In particular, the electric field decreases the number of particles escaping to the wall along superbanana orbits (within about $1 \mathrm{~ms}$ ) and increases the fraction of transitioning particles that are confined or lost for $\Delta t \gg 1 \mathrm{~ms}$ due to stochastic diffusion. This conclusion agrees with a recent publication [21] where the influence of the negative neoclassical electric field on NBI ions in W7-X was studied. It was found that the confinement of partly sloweddown NBI ions is considerably improved due to this field. A similar improvement in the confinement of locally trapped particles was also predicted in earlier work [18]. Later it 
was however shown that the electric field arising from neoclassical transport in W7-X is not sufficient to drastically improve the confinement of NBI ions [19].

The presence of this electric field actually simplifies the trapped-to-passing conversion of NBI ions in W7-X. According to figure A2, no fast ions with fully trapped (non-transitioning) orbits will be produced by NBI in the first NBI experiments using the high-mirror configuration. The birth orbits of the injected ions will be either fully passing or fall in the region of transitioning particles. Most of the ions in the latter group have $\lambda$ close to 0.9 . Those of them that are born in the locally trapped state are shown in figure A1 by thin lines. It follows from the left panel of this figure (valid for $\left.E_{0 r}=0\right)$ that most particles in the locally trapped state do not reach the separatrix $\kappa^{2}=1$ but escape to the wall. In contrast, we observe in the right panel of figure A1 that most of the thin lines intersecting the equatorial plane reach the separatrix. If the radial profile of the NBI ions is peaked in the core, this implies that most particles born in the locally trapped state become locally passing (within about $1 \mathrm{~ms}$ ). It thus follows that in order to convert trapped particles into passing ones, it is sufficient to provide interaction with the RF field when they are in the locally passing state.

\section{Acknowledgments}

This work was supported in part by the Project No. 6058 of the Science and Technology Center in Ukraine and the National Academy of Sciences of Ukraine (NASU) and the Project No. 0114 U000678 of NASU.

\section{Appendix A. Trapped energetic ions in the first planned W7-X experiments with NBI}

In fully optimized Wendelstein-line (so-called quasi-isodynamic) stellarators, the contours of constant magnetic field are closed poloidally everywhere except for the near-edge region. However, this will be not the case in the initial stage of operation with NBI in W7-X, where there are unclosed contours of $B_{0}=$ const going through the plasma core, see figure 6 . A considerable fraction of fast ions may therefore quickly escape to the wall, moving along superbanana orbits, even when their pitches lie in the region of transitioning particles. Figure A1, left panel, demonstrates this behavior. In this figure the pitch parameter $\alpha\left(\alpha \equiv \lambda^{-1}-1\right)$ was taken equal to $0.1(\lambda=0.9)$, which corresponds to the maximum of the $\lambda$-distribution of the injected ions at mid-plasma radius, see figure A2. In spite of the fact that this pitch lies in the region of transitioning particles, most fast ions with this pitch, for $\Delta t \sim \tau_{s b}$, quickly escape from the plasma. (Only a small number of particles are really transitioning, i.e., such particles that are transformed to locally passing and back again, intersecting the curve $\kappa^{2}=1$ ). The magnitude of $\tau_{s b}$ can be estimated as $\tau_{s b}=2 \pi r / v_{p r}$, where $v_{p r}=0.5 \rho v \partial \ln B_{0} / \partial r$ is the poloidal precession velocity, which gives $\tau_{s b} \lesssim 1 \mathrm{~ms}$. This estimate agrees with the results of numerical calculations in [3], where a considerable loss of fast ions was 

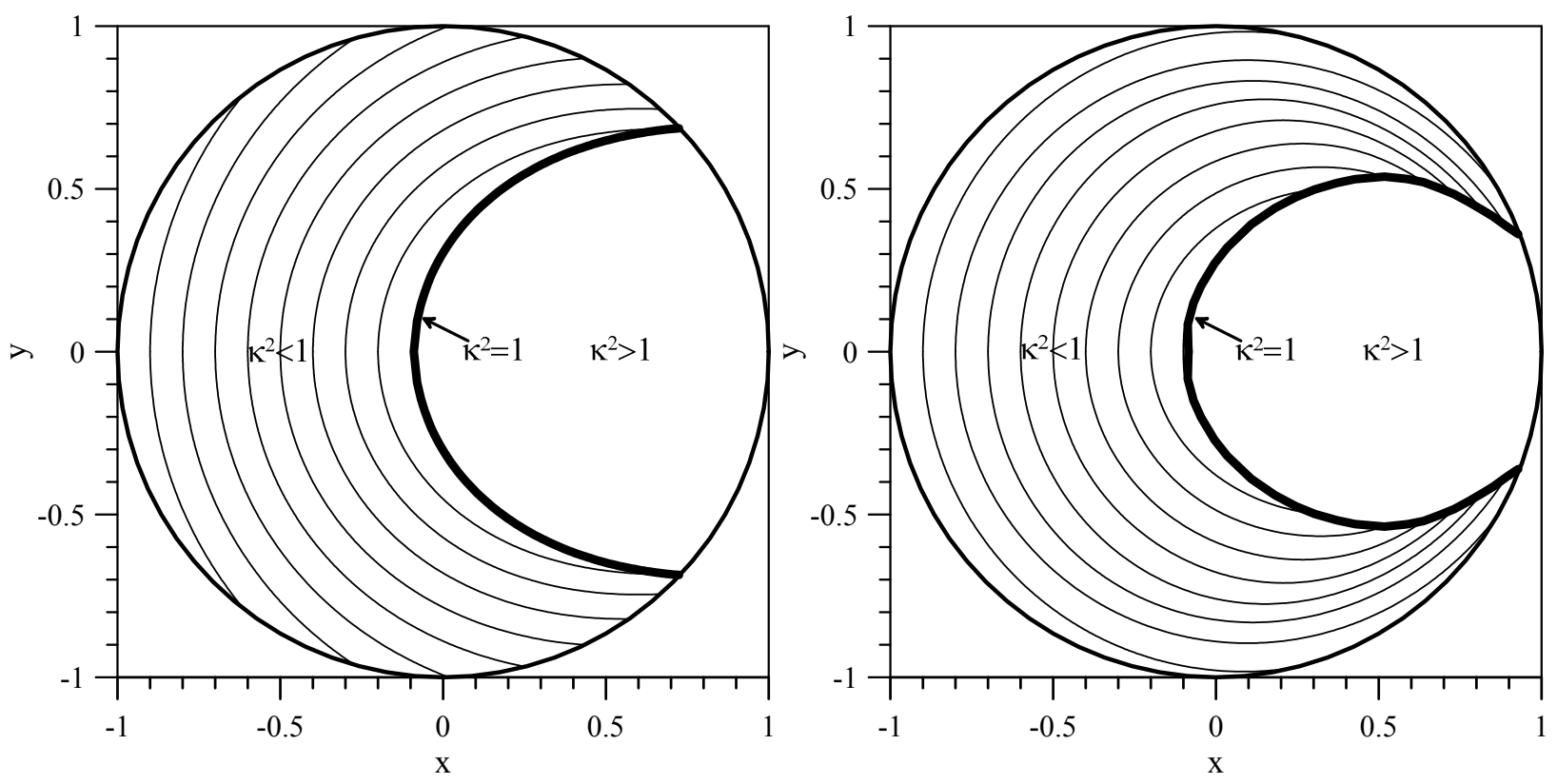

Figure A1. The trapping parameter $\kappa^{2}=1$ (bold line) and contours of $\mathcal{J}=$ const for $\alpha=0.1$, i.e., $\lambda=0.9$ (thin lines) in the toroidal plasma cross section for the W7-X high mirror configuration when $E_{0 r}=0$ (left panel) and $E_{0 r} \neq 0$ (right panel). The latter is calculated under the assumption that $\Phi_{0}(r)=-\int_{r_{0}}^{r} d r^{\prime} E_{0 r}\left(r^{\prime}\right)$, with $r_{0}$ a point at the axis $x_{0}<0, y=0$. This implies that the case with $E_{0 r} \neq 0$ holds for particles launched at $x<0, y=0$. Here $E_{0 r}<0$ is the neoclassical equilibrium electric field (shown in figure B1 of reference [19]). The major radius of the torus is located to the left. The trapping parameter $\kappa^{2}(r, \vartheta)$ in the presence of the electric field is defined in reference [18].

observed for $\Delta t \lesssim 1 \mathrm{~ms}$. One can hope, however, that in reality the confinement of fast ions with $\lambda=0.9$ will be better thanks to the radial electric field, as follows from figure A1, right panel. This field is set by the requirement that the neoclasscical flux must be ambipolar, even in the presence of gyrokinetic turbulence [22]. We observe that many $\mathcal{J}=$ const contours reach the $\kappa^{2}=1$ curve rather than the plasma boundary. This means that these particles really become transitioning and, therefore, their confinement time increases. Furthermore, calculations show that the separatrix $\kappa^{2}=1$ of some particles with smaller $\alpha$ does not cross the plasma edge, in which case any stochastic diffusion results in radial transport, but not loss to the wall.

\section{References}

[1] Helander P. 2014 Rep. Prog. Phys. 77087001 (35pp)

[2] Lotz W., Merkel P., Nührenberg J., and Strumberger E. 1992 Plasma Phys. Control. Fusion 34 1037

[3] Drevlak M., Geiger J., Helander P., Turkin Y. 2014 Nucl. Fusion 54073002

[4] Beidler C. D., Harmeyer E., Herrnegger F., et al. 2001 Nucl. Fusion 411759

[5] Subbotin A.A, Mikhailov M.I., Shafranov V.D., Isaev M.Yu., Nührenberg C., Nührenberg J., Zille R., Nemov V.V., Kasilov S.V., Kalyuzhnyj V.N., Cooper W.A., 2006 Nucl. Fusion 46921 


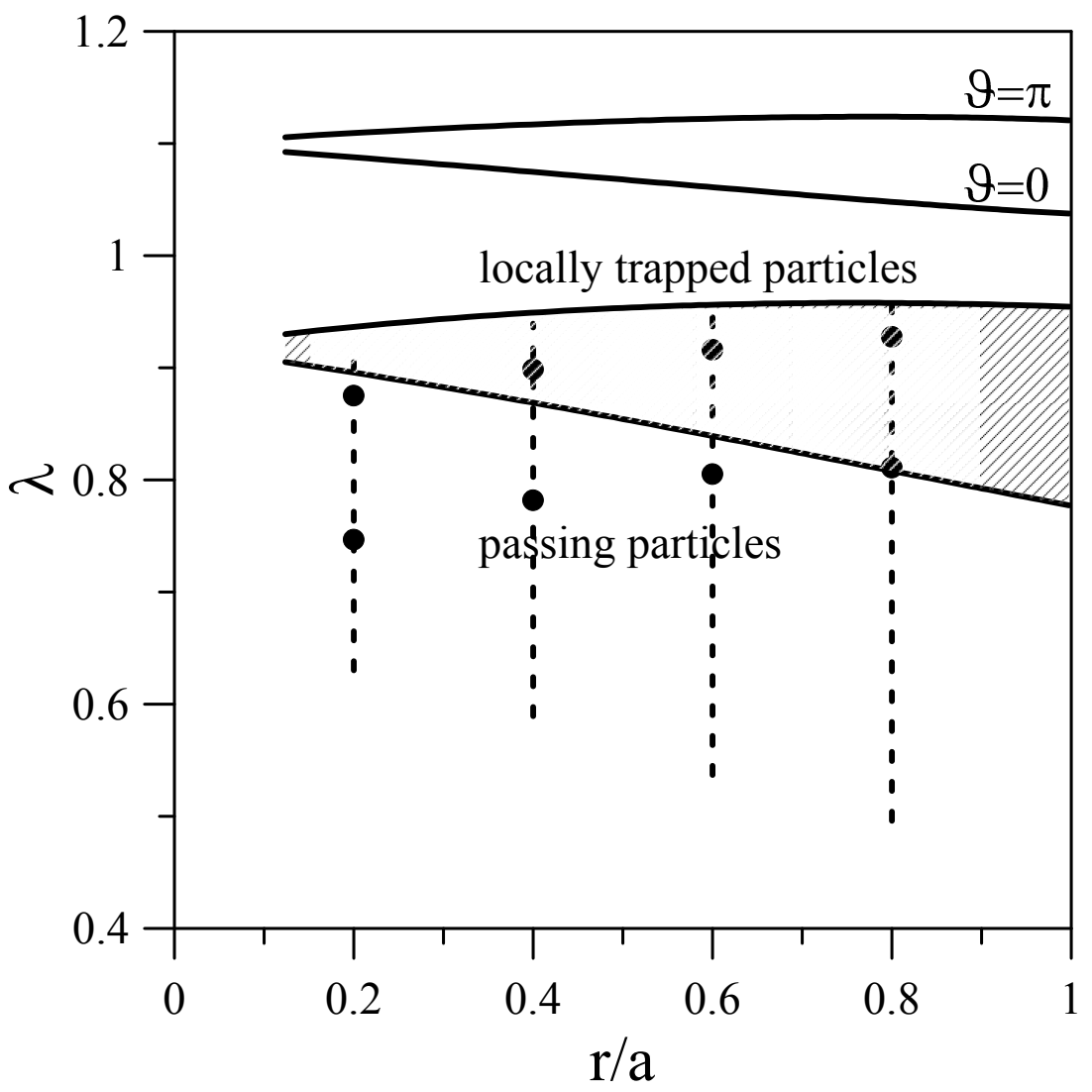

Figure A2. Pitch parameter $(\lambda)$ at several radii of the NBI produced fast ions (vertical dotted lines) in the plane showing regions of locally trapped particles, transitioning particles, and passing particles in the W7-X high mirror configuration. The region of transitioning particles is shadowed. The range of $\lambda$ where most ions are born is shown on each vertical dotted line by solid circles. The lowermost solid line represents the trapped-passing boundary, which is independent of $\vartheta$.

[6] Helander P., Nührenberg J. 2009 Plasma Phys. Control. Fusion 51055004

[7] Beidler C. D., Kolesnichenko Ya. I., Marchenko V. S., Sidorenko I. N., Wobig H. 2001 Phys. Plasmas 82731

[8] Stix T.H. 1992 Waves in Plasmas, Springer-Verlag New York, Inc.

[9] Belikov V.S., Kolesnichenko Ya.I. 1982 Plasma Physics 2461

[10] Eriksson L.-G, Helander P. 1994 Phys. Plasmas 1308

[11] Kolesnichenko Ya. I., White R.B., Yakovenko Yu.V. 2002 Phys. Plasmas 92639

[12] Mantsinen M.J., et al. 2002 Phys. Rev. Lett. 88105002

[13] Kolesnichenko Ya. I., Könies A., Lutsenko V. V., Yakovenko Yu.V. 2011 Plasma Phys. Control. Fusion 53024007

[14] Kolesnichenko Ya. I., Lutsenko V. V., Weller A., Werner A., Wobig H., Yakovenko Yu.V. Geiger J., Zegenhagen S. 2006 Nucl. Fusion 46753

[15] Ongena J., et al. 2014 Phys. Plasmas 21061514

[16] Helander P., Lisak M., 1992 Phys. Fluids B 41927

[17] Belikov V.S., Kolesnichenko Ya. I. 1994 Plasma Phys. Control. Fusion 361703

[18] Kolesnichenko Ya. I., Lutsenko V. V., Tykhyy A. V., Weller A., Werner A., Wobig H., Geiger J 2006 Phys. Plasmas 1307250

[19] Kolesnichenko Ya. I., Könies A., Lutsenko V. V., Drevlak M., Turkin M., Helander P. 2016 Nucl. Fusion 56066004 
[20] Park H.K. et al. 2015 42nd EPS Conference on Plasma Physics, Report P4.151

[21] Faustin J.M., Cooper W.A., Graves J.P., Pfefferle D., and Geiger J. 2016 Nucl. Fusion 56092006

[22] Helander P., Simakov A.N., 2008 Phys. Rev. Lett 101145003 